\title{
More than Academic Competence: the Importance and Utility of Internship to Gain Clinical and Research Experiences
}

\author{
Izabela C. Leahy ${ }^{1} \cdot$ Amanda W. Baier $^{1} \cdot$ Paul R. Hickey $^{1}$
}

Published online: 26 July 2018

(C) The Author(s) 2018

\section{Introduction}

Globalization, information technology, and advancements in science are shaping new realms of knowledge sharing and communication in the twenty-first century. This accelerated process has a significant impact on education and teaching models and, consequently, poses a challenge for colleges and universities given their responsibility for cultivating the skills of the future workforce. In his book, College (Un)Bound, Selingo argues that high-impact practices, such as undergraduate research experiences, internships, and study abroad opportunities, will become progressively more important in this evolving learning environment [1], as students aim to distinguish themselves in an increasingly competitive graduate school application and job market.

In order to provide students interested in healthcare-related careers with the opportunity for this essential high-impact practice and critical interpersonal development, programs with an innovative pedagogy curriculum that is inclusive of role modeling, exemplified professionalism, together with comprehensive and expert understanding of clinical research and scientific discovery, must be made available. For those students seeking acceptance to medical and other graduate schools, the need to participate in these "high-impact practices" is even greater. Admissions committees and educators agree that success in medical school requires more than academic competence [2]. Indeed, successful candidates not only have near-perfect GPAs and stellar MCAT scores, but they possess experiences and attributes that demonstrate integrity, altruism, self-management, interpersonal and teamwork skills, and other characteristics [3, 4].

Izabela C. Leahy

Izabela.Leahy@childrens.harvard.edu

1 Department of Anesthesiology, Critical Care and Pain Medicine, Boston Children's Hospital, Harvard Medical School, 300 Longwood Ave, Boston, MA 02115, USA
American theorist David A. Kolb believes that "learning is the process whereby knowledge is created thought transformation of experience" [5]. The Pediatric Anesthesia Clinical and Research Internship (PACaRI) within the Department of Anesthesiology, Critical Care, and Pain Medicine at Boston Children's Hospital was designed with these specific objectives in mind, by targeting the academic interests and ambitions of high school, undergraduate, and graduate students. The PACaRI provides a learning environment that is both exciting and challenging, one that empowers both students and involved faculty to pursue learning. It aims to engage students to become compassionate and caring clinicians and healthcare leaders by challenging them to think critically about research, scientific discovery, ethics, safety, efficacy, and efficiency of healthcare delivery systems. PACaRI is composed of (1) introduction to research, followed by direct participation in a retrospective research project; (2) participation and presentation of research at national conferences; (3) hands-on clinical work in the operating rooms; and (4) participation in lectures and discussion-based group meetings with targeted academic and career advisement.

This manuscript describes the design, importance, and impact of PACaRI and highlights the need and demand for the adoption of similar programs at other institutions.

\section{PACaRI Background}

Since its inception in 2010, the Pediatric Anesthesia Clinical and Research Internship (PACaRI) has been nurturing and educating a growing fraction of the next generation of scientists, researchers, and healthcare leaders. A total of 87 interns from 24 institutions have graduated or are currently participating in the Program. Twenty-nine faculty members have participated as mentors. The summer internship opportunity lasts for 13 weeks, while the extended internship lasts approximately 32 weeks. Mentorship is provided both one-to-one and in a group setting. Each participant's mentor is an experienced 
medical professional, providing advice on admissions to medical schools and other fields in healthcare professions and humanities, as well as professional and personal development. Since year three of the Program, the number of applications has increased by over $1000 \%$. In 2017 , the acceptance rate was $9 \%$. Table 1 provides demographic information of the accepted interns.

PACaRI applies "Situated learning" theory that provides particularly powerful models of how professionals learn to apply technical knowledge within innately varied social contexts [6-9]. This perspective suggests that "learning to do" (closely related to "knowing how") takes place through solving problems in context. Crucially, the context contains other people who are experienced at solving similar problems. Theoretical descriptions of the learning process at work differ within this perspective [8], but all essentially have novices learning a holistic set of actions and explanations from experienced practitioners. Novices learn what to observe, what interpretations to link to observations, and what words and actions to use when conveying these both to clients and to colleagues. Novices learn best to apply the technical knowledge within skilled actions (e.g., clinical decision-making) in rich, relevant contexts. This context reinforces the developing professional identity of the learner; this is how real professionals [school teachers/doctors] behave with real clients [children/patients] and is therefore highly motivational [6].

\section{Introduction to Research: Retrospective Research Project and Presentations of Findings}

The primary focus of the Program is the early introduction of its participants to the importance and implications of clinical research. Cohen and Manion distinguished research as being systematic, controlled, built on inductive-deductive reasoning,

Table 1 Demographic information of the interns, 2010-2017

\begin{tabular}{lcc}
\hline Total $N=87$ & Number & Percent (\%) \\
\hline Gender & 35 & 40 \\
Male & 52 & 60 \\
Female & \\
Level of education at program acceptance & 49 & 56 \\
Graduate & 34 & 39 \\
Undergraduate & 4 & 5 \\
High School & & \\
Race & 18 & 21 \\
Asian & 8 & 9 \\
Black or African American & 0 & 0 \\
Native Hawaiian or other Pacific Islander & 54 & 62 \\
White & 5 & 6 \\
Hispanic & 2 & 2 \\
No data & & \\
\hline
\end{tabular}

and empirical (i.e., related by observation of "objective reality") [10]. To accomplish this, students are paired with a Department faculty member for the duration of their internship, who serves as the Principal Investigator (PI) on the hypothesis-driven retrospective research study. Each student pursues a focused question in depth with close guidance from a faculty member. This partnership facilitates mentoring opportunities for the PI, which proves especially important for getting interns interested in academic careers [11] and certain specialties or sub disciplines, since those with mentors in these areas experience more opportunities for research $[12,13]$. In order to obtain career and psychosocial benefits from participating in a mentoring relationship, the protégé must interact with the mentor to discuss problems, set personal and workrelated goals, and obtain guidance on career and personal issues $[14,15]$. Also, the protégé must effectively utilize the time spent with the mentor by asking questions and discussing problems and interests in order to obtain the full range of benefits available from the relationship.

Program leaders and PIs provide explicit instruction and offer a wealth of experience in key elements of clinical research processes; under their direction and mentorship, interns are involved in protocol submission, scientific and institutional review, data collection, management, analysis, and generation of findings. PIs and Program leaders encourage students to interact with knowledge - to critique and manipulate, design, create, and transform it. This means valuing prior knowledge and experience; promoting learner responsibility through facilitating rather than directing learning; encouraging learners to test out and apply new knowledge; and using small-group work to foster the elusive skills of critical thinking and reflection [16].

Research work teaches interns persistence and perseverance, particularly when faced with rejection, roadblocks, and challenges. Additionally, research experience provides interns with the knowledge necessary to excel in science studies, differentiating them from those who only gain it from textbooks. While emphasis is placed on ensuring that students have a thorough understanding of the research process and accompanying ethical considerations, the ultimate aim is to provide research opportunities including acknowledgement of participation, and rewards for achievement. To encourage active student participation and to recognize their active role in the discovery process, the reward is presentation of the research project to local and national leaders in the field. At the completion of PACaRI, students present their research findings to faculty members during Anesthesia Grand Rounds. This forum provides the opportunity to field questions from attendees, forcing them to think critically about their work and serves as preparation for those presenting projects at national and international conferences.

The interns' research accomplishments are measured by the number of scholarly achievements. Since the beginning 
of the Program $87(100 \%)$, interns presented at PACaRI Ground Rounds, $70(80 \%)$ at the Department's Ground Rounds, while 26 (30\%) interns presented 32 abstracts at various professional conferences around the country. Six interns (7\%) have been included as co-authors on publications.

\section{Clinical Experience: Hands-on Clinical Work in the Operating Rooms}

One of the more critical experiences for interns is exposure to patients in a clinical setting. For interns interested in pursuing careers in medicine, this type of experience often serves to reinforce that desire. For interns without previous clinical experience, this exposure allows them to determine if this aspect of healthcare is appealing to them. Through their work in the operating rooms, interns can gain greater clarity about future career and educational goals [13].

The design and structure of this aspect of PACaRI is unique, as interns are introduced to the fast-paced, multidisciplinary operating room environment, through observation of varied surgical procedures; interns also perform hands-on clinical work by assisting the Department's anesthesia technician team.

In this supportive learning environment, all involved parties are able to hone their skill, which increases engagement and efficiency of work and therefore becomes positive for the institution as a whole $[11,17,18]$. Interns in the PACaRI have the unique opportunity to closely observe procedures while building a relationship with their mentor in the field, and asking questions as they arise. Shadowing a mentor in this capacity allows the intern to gain insight about what it is like to work at varying levels of power in an organization [17]. They learn about the expectations, challenges, and rewards involved in the practice of medicine, as well as gain a deeper understanding of the importance of teamwork during surgery. Additionally, observation in the OR provides many students with greater clinical context for their research projects.

\section{Educational Experience: Targeted Academic Lectures and Career Advisement}

The driving principle of PACaRI is that today's students are active learners rather than spectators; it encourages students to view themselves as participants in creating information and new ideas ${ }^{18}$. Rethinking pedagogy for the twenty-first century is as crucial as identifying the new competencies that today's learners must develop. Traditional approaches emphasizing memorization or the application of simple procedures will not advance learners' critical thinking skills or autonomy. To develop the higher-order skills they now require to be successful in both their educational pursuits and careers, individuals must engage in meaningful inquiry-based learning that has genuine, recognized value and relevance for them personally and in their communities.

In addition to extensive research and clinical opportunities, PACaRI also provides participants with avenues for learning and education by incorporating into the internship curriculum a series of workshops and seminars presented by Department faculty members and other experts. Additionally, all students are invited to attend Grand Rounds, held on a weekly basis, to explore specific anesthesia, surgical, and pertinent hospitalrelated topics. The topics presented cover important issues related to research, medical ethics, and discovery in healthcare today.

As of November 2017, the status of interns is as follows: $2.5 \%$ in residency programs, $41 \%$ in medical school, $13 \%$ in graduate school, $17 \%$ in undergraduate school, $24 \%$ working in healthcare, and $2.5 \%$ were lost to follow-up.

\section{Discussion}

Evaluation of the Program suggests that it serves to provide participants with unique experiences and opportunities that, coupled with their education, help to better prepare them for entrance into medical school and other chosen higher level education opportunities in the healthcare industry. PACaRI participants report having a competitive edge in the medical school admissions process; while medical admission committees look closely at applicants' MCAT scores and undergraduate GPAs, PACaRI interns can enhance their personal statements and include letters of recommendation that speak to professional/healthcare experience.

PACaRI interns' experiences include those described by both Frei et al. and von der Borch et al. such as career counseling, creating student-faculty connections [12, 19], and promoting and monitoring professionalism [11, 13]. First published in 2011, the book "Intern Nation" by Ross Perlin documents and analyzes the rise of internships in the modern workplace, the economic impact of internships, the social inequalities they perpetuate, and their effect on particular industries and rite of passage for young people [20]. According to Perlin, the first interns were doctors; one of the earliest mentions is in a report to the trustees of a Boston hospital in 1865. Perlin's study estimates that $75 \%$ of students at four-year colleges intern at least once. While a growing number of American colleges and universities have made participation in an internship a condition of graduation, some internships are not viewed as valuable [20]. As demonstrated by the increase in the PACaRI application rate, the demand and interest in participation has increased dramatically. In 2017, we received 170 applications for 16 internship opportunities which resulted in a $9 \%$ acceptance rate. While the increased numbers of applications along with the reported benefits from participants are suggestive of the Program success, there is no 
control group to use for comparison. To better examine and demonstrate the value of the Program, evaluations are completed by students to provide additional means for assessment. Further evaluation and analyses are underway to demonstrate the benefits of the Program to the Department.

In the years since its inception, PACaRI has evolved, restructured, and grown into the Program it is today. This evolution has not come without its challenges, many of which Program leadership has been able to address and overcome. One of the challenges encountered has been partnering students with Principal Investigators and research protocols which they are able to complete during their time in the Program. Ideally, research protocols are well-defined, with clear aims and electronically available data. In our experience, students' involvement in prospective research protocols may provide a broad understanding of the clinical research process; however, due to the nature of these studies, interns are unable to participate in the development of abstracts and manuscripts. Another ongoing challenge has been the inconsistency with which interns raise questions and communicate their needs. To foster open communication, Program Leadership holds consistent and frequent check-ins and meetings with interns and has implemented online evaluations that are disseminated at numerous times throughout the internship. Real-time discussion of concerns or uncertainties allows Leadership to take action when necessary and helps to ensure students' productivity and success.

As leaders of PACaRI, we recognize the importance of striking a balance between the standard learning environment, knowledge acquisition, and research experience with ongoing willingness and ability for reflection and critical analysis. PACaRI's integration of the clinical and research disciplines, supplemented by substantive educational opportunities, offers learning through scientific exploration and the acquisition of clinical skills. It is our hope that these experiences shape the development and engagement of future clinicians and healthcare leaders by challenging them to become critical thinkers and problem-solving, professional learners.

Acknowledgements The authors would like to thank Vanessa Young, RN, BA, for her assistance managing and developing the Program during her time in the Department.

\section{Compliance with Ethical Standards}

Conflict of Interest The authors declare that they have no conflict of interest.
Open Access This article is distributed under the terms of the Creative Commons Attribution 4.0 International License (http:// creativecommons.org/licenses/by/4.0/), which permits unrestricted use, distribution, and reproduction in any medium, provided you give appropriate credit to the original author(s) and the source, provide a link to the Creative Commons license, and indicate if changes were made.

\section{References}

1. Selingo J. College (un)bound: the future of higher education and what it means for students. Houghton Mifflin Harcourt: New York; 2013.

2. Albanese M, Snow M, Skochelak S, Huggett K, Farrell P. Assessing personal qualities in medical school admissions. Acad Med. 2003;78:313-21.

3. Adams K, Goodwin G, Searcy C, Norris D, Oppler S. Development of a performance model of the medical education process. Washington, DC: The American Institutes for Research; 2001.

4. Carrothers R, Gregory S, Gallagher T. Measuring emotional intelligence of medical school applicants. Acad Med. 2000;75:456-63.

5. Kolb DA. Experiential learning: experience as the source of learning and development. Englewood Cliffs: Prentice-Hall, Inc.; 1984.

6. Lave J, Wenger E. Situated learning \pm legitimate peripheral participation. Cambridge: Cambridge University Press; 1991.

7. Billett S. Situated learning: bridging sociocultural and cognitive theorising. Learn Instr. 1996;6(3):263-80.

8. Chaiklin S, Lave J, editors. Understanding practice: perspectives on activity and context. Cambridge: Cambridge University Press; 1993.

9. Brown J, Collins A, Duguid P. Situated cognition and the culture of learning. Educ Res. 1989;18:32-42.

10. Cohen L, Manion L. Research methods in education. 4th ed. London: Routledge; 1994.

11. Fornari A, Murray T, Menzin A, Woo V, et al. Mentoring program design and implementation in new medical schools. Med Edu Online. 2014.

12. Frei E, Stamm M, Buddeberg-Fischer B. Mentoring programs for medical students - a review of the PubMed literature 2000-2008. BMC Med Educ. 2010;10:32.

13. Dimick C. Professional practice experience. Internships open the door to real-world lessons. J AHIMA. 2009;80(10):38-42.

14. Kram K. Mentoring at work: developmental relationships in organizational life. Scott Bresman: Glenview; 1985.

15. Kram K, Isabella L. Mentoring alternatives: the role of peer relationships in career development. Acad Manag J. 1985;28:110-32.

16. Maudsley G, Strivens J. Promoting professional knowledge, experiential learning and critical thinking for medical students. Med Educ. 2000;34:535-44.

17. Ragins B, Scandura T. Burden or blessing? Expected costs and benefits of being a mentor. J Organ Behav. 1999;20(4):493-509.

18. Hunt D, Michael C. Mentorship: a career training and development tool. Acad Manag Rev. 1983;8(3):475-85.

19. von der Borch P, Dimitriadis K, Störmann S, et al. A novel largescale mentoring program for medical students based on a quantitative and qualitative needs analysis. GMS Z Med Ausbild. 2011.

20. Perlin R. Intern nation: how to earn nothing and learn little in the brave new economy. Brooklyn: Verso Books; 2011. 